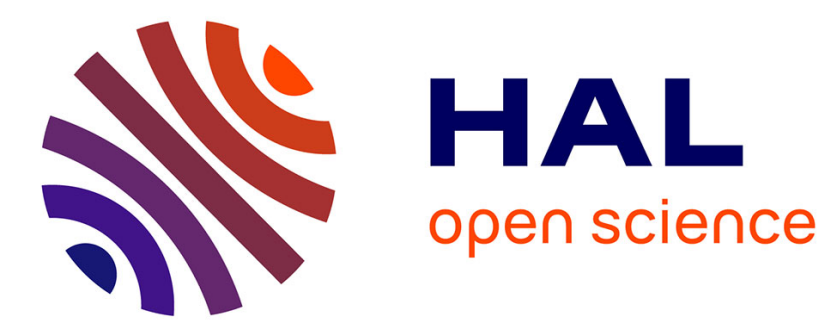

\title{
Entre l'épistémologie et la communication Eliseo Veron
}

\section{To cite this version:}

Eliseo Veron. Entre l'épistémologie et la communication. Hermès, La Revue - Cognition, communication, politique, 1997, 21, pp.25-32. halshs-01487123

\section{HAL Id: halshs-01487123 https://shs.hal.science/halshs-01487123}

Submitted on 10 Mar 2017

HAL is a multi-disciplinary open access archive for the deposit and dissemination of scientific research documents, whether they are published or not. The documents may come from teaching and research institutions in France or abroad, or from public or private research centers.
L'archive ouverte pluridisciplinaire HAL, est destinée au dépôt et à la diffusion de documents scientifiques de niveau recherche, publiés ou non, émanant des établissements d'enseignement et de recherche français ou étrangers, des laboratoires publics ou privés. 


\section{ENTRE L'ÉPISTÉMOLOGIE ET LA COMMUNICATION}

Les sciences constituent, en premier lieu, un ensemble de faits institutionnels. De ce point de vue, être chercheur (ou enseignant chercheur, comme on dit dans les milieux universitaires) est une profession parmi d'autres, mais une profession non libérale : on fait de la science à l'intérieur d'un projet institutionnel à caractère collectif. Une formation longue amènel'individu qui a choisi ce métier à obtenir une légitimité professionnelle précise, mais contrairement aux avocats, aux médecins ou aux architectes, son activité devra s'exercer au sein d'une communauté, avec d'autres chercheurs. En France, à la différence d'autres pays industriels, le statut du chercheur scientifique est encadré par l'État : le chercheur ou l'enseignant chercheur est le plus souvent un fonctionnaire. Mais indépendamment du fait que son statut soit défini dans le cadre de l'espace public ou dans celui d'une logique du privé, les institutions scientifiques donnent lieu à des phénomènes organisationnels comparables à ceux d'une entreprise : normes collectives qui définissent les objectifs de l'organisation, problématique de recrutement et de ressources humaines, infrastructure technologique des laboratoires, hiérarchie de pouvoir, logique budgétaire, gestion administrative, contrôle permanent de la qualité du travail effectué.

Ce parallèle avec l'entreprise industrielle parait d'autant plus pertinent que la dimension productive semble inséparable de la notion de science. Dans un bel article, Emile Benveniste avait étudié la genèse de l'adjectif scientifique (Benveniste, 1974, p. 247-253) en soulignant le fait que les dérivés en -fique ne sont jamais «de simples adjectifs de relation». Si le substantif «science » avait produit un adjectif de simple relation, celui-ci aurait dû être « sciential » ou «scientique ». Les adjectifs en -fique comportent, remarque Benveniste, une fonction «factitive » qui leur est essentielle. Ainsi calorifique, frigorifique, soporifique (qui produit la chaleur, le froid, le sommeil) ou bien « honorifique » (qui procure l'honneur). Ce qui est « scientifique », c'est ce qui produit du savoir.

De ce point de vue les institutions scientifiques seraient des ensembles organisationnels complexes axés sur le processus de production d'un produit : la connaissance. Or, normalement, 
les produits d'une entreprise industrielle sont destinés soit à d'autres entreprises qui vont encore les transformer ou les incorporer dans d'autres produits, soit directement à la société, c'est-àdire, au marché de la consommation. Qui sont les « consommateurs » de ces produits particuliers que l'on appelle les « connaissances scientifiques » ? C'est l'une des questions essentielles qui nous intéressent ici.

Un destinataire évident des produits que l'on appelle des connaissances scientifiques ce sont précisément les industriels : à partir de ces connaissances, ils vont fabriquer des microprocesseurs, par exemple, qui feront partie, en fin de course, des ordinateurs que l'on pourra acheter sur le marché. C'est la transformation de la connaissance scientifique en innovations technologiques.

Mais une particularité des institutions scientifiques qui les différencie, peut-être, d'une entreprise industrielle, c'est que les premiers destinataires des connaissances scientifiques semblent être les scientifiques eux-mêmes : on dirait qu'il y a un mécanisme endogène particulier par lequel ces usines de production de connaissances que sont les institutions scientifiques se nourrissent tout d'abord d'elles-mêmes., Autrement dit, on ne peut produire de nouvelles connaissances qu'à partir des connaissances que l'on vient de produire. On pourrait aller jusqu'à dire que le destinataire privilégié des connaissances scientifiques produites par un chercheur ce sont ses collègues, les autres chercheurs qui travaillent dans le même secteur que lui.

Il y aurait donc une caractéristique propre des institutions scientifiques : cette étrange autarcie par laquelle ce qu'elles produisent constitue leur nourriture première. C'est un peu comme si, par exemple, le destinataire privilégié des informations produites par un journaliste étaient les autres journalistes, et non pas le public qui consomme les médias d'information.

Pour voir plus clair, essayons de préciser ce qu'est ce produit que nous avons appelé connaissance scientifique. Or, justement ici un autre parallèle devient étonnamment pertinent : une institution scientifique ressemble somme toute à un média, plus spécifiquement, à une institution médiatique d'information, par exemple, à une chaîne de télévision dans sa fonction informative. Quel est l'input, dans un cas et dans l'autre, qui met en marche le processus de production ? Des informations, sous forme de discours. Quel est l'output, après l'utilisation de machines plus ou moins sophistiquées ? Des informations, sous forme de discours. Dans un cas comme dans l'autre, les discours à l'entrée et à la sortie ne sont pas identiques : il y a eu, entre l'entrée et la sortie, des transformations. Et aussi bien l'institution scientifique que l'institution médiatique informative disent nous parler d'un réel, que nous appelons «le monde ».

On peut penser que ce parallèle n'est pas entièrement satisfaisant, à partir de l'intuition selon laquelle le discours médiatique de l'information et le discours scientifique n'ont pas le même statut. On peut faire valoir, par exemple, que le discours qualifié de «scientifique », comme nous l'avons rappelé tout à l'heure à propos de la genèse linguistique de cet adjectif, est ce discours où l'on produit du savoir, tandis que le discours de l'information à la télévision ou dans la presse, ne fait que transmettre des informations, sans produire véritablement une nouvelle connaissance ou un nouveau savoir. Et pourtant, cela se discute. J'ai commis un livre, il 
y a quelques années, où j'essayais de montrer que les médias informatifs ne reproduisent ni ne reflètent rien : ils produisent le réel social dont ils parlent (Véron, 1981). Il y aurait alors, dans les deux cas, une dimension productive importante.

Faut-il donc, pour prolonger ce parallèle, dire que l'activité scientifique, comme l'activité médiatique de l'information, produit la réalité sur laquelle elle travaille, par opposition à l'idée selon laquelle la science ne fait que découvrir les lois d'un réel qui est là, indépendamment du discours que l'on tient sur lui ? Cette dernière idée, qui est celle du « réalisme » (plus ou moins naif selon le point de vue) s'oppose à l'hypothèse constructiviste de la production du réel par la connaissance : la nature que nous connaissons à travers le savoir scientifique ne serait pas la nature en soi, mais la nature telle que nos catégories et nos mécanismes cognitifs nous permettent de la constituer. Sur cette polémique, à proprement parler épistémologique, nous ne nous attarderons pas. Elle dépasse, bien entendu, la portée de cet article.

En éludant la polémique entre réalistes et constructivistes en ce qui concerne la connaissance scientifique proprement dite, contentons-nous de signaler que la plupart des spécialistes en épistémologie de la science s'accorderaient à dire que les objets qu'étudie la science, ne se trouvent pas comme cela, déjà constitués, comme on trouve des arbres et des pierres. Les objets de la science se construisent (quelle que soit la portée exacte que l'on donne à ce terme) à travers des ensembles extrêmement complexes de concepts, de théories, des dispositifs techniques d'observation et de mesure, sans lesquels il n'y aurait pas à proprement parler d'objet à étudier. Jusqu'ici, notre parallèle semble rester valable : sans les dispositifs complexes qui constituent cette institution que nous appelons une chaîne de télévision, il n'y aurait pas ce que nous appelons, aujourd'hui, l'« actualité ».

$\mathrm{Si}$ nous croisons maintenant les deux parallèles que nous avons suggérés (celui entre institution scientifique et entreprise industrielle, d'une part, et celui entre institution scientifique et média d'information d'autre part) nous pourrons peut-être avancer. Pour ce qui est du deuxième parallèle, il est clair qu'il existe une différence importante entre les deux institutions. Bien entendu, la question de savoir si l'information est aujourd'hui ou non à proprement parler un produit, une marchandise, n'est pas une mince interrogation. Nous y reviendrons. Mais il est clair en tout cas que si l'on veut appliquer cette notion de marchandise, il s'agit d'une marchandise grand public, comme on dit : l'institution médiatique fait circuler, diffuse cette marchandise (l'information sur l'«actualité » du monde) directement sur le marché, elle est destinée à chacun de nous en tant que citoyen, comme n'importe quel produit de grande consommation. Malgré de multiples médiations, le destinataire final de l'activité industrielle de nos sociétés est le marché de la consommation. Alors que le produit « connaissance scientifique » ne semble pas, justement, avoir a priori une telle destination : nous revenons, sous un autre angle, à la question du caractère endogène de cette connaissance, qui semble être destinée, tout au moins au prime abord, à la même communauté restreinte qui l'a produite.

Cet aspect de clôture de la production des connaissances scientifiques nous permettra, peut-être, de cerner une différence spécifique de l'activité scientifique aujourd'hui. Elle n'appa- 
raît pas du point de vue de la production, mais plutôt du point de vue de la réception. Du point de vue de la production, toute activité spécialisée suppose, bien entendu, l'acquisition d'un savoir-faire lui même spécialisé. La formation pour devenir architecte est longue et spécifique ; elle est longue et spécifique aussi pour devenir spécialiste en physique des particules. Par ailleurs, une tendance à la clôture, ou plutôt à l'autonomisation progressive, est caractéristique du fonctionnement et de l'évolution historique de toutes les corporations professionnelles.

Mais l'avocat qui s'occupe de mes affaires, l'architecte qui construit ma maison, le médecin qui me traite à l'hôpital rentrent, s'insèrent, à un moment ou à un autre, dans l'interface typique d'une prestation de services professionnel/citoyen (ce dernier ayant le statut d'un client) caractéristique des professions dites libérales. Alors que le simple citoyen n'a aucun service à attendre du spécialiste en physique des particules; il ne saurait même pas formuler une quelconque demande à son égard. Et si, à propos du physicien des particules, l'on posait la question de savoir pour qui il travaille?, on serait tenté de répondre : pour lui-même et pour ses collègues qui font, eux aussi, de la physique des particules. Le bénéficiaire social de son activité, autre qui lui-même et ses semblables n'eșt pas donné, n'est pas identifiable d'emblée dans le contexte social.

Nous pouvons donc soupçonner, comme hypothèse de travail, qu'il y a quelque chose dans la profession de chercheur scientifique, qui le différencie des autres professions. L'architecte, l'avocat, le notaire ne sont nullement perçus par la société comme des chercheurs : même si leur activité repose sur des savoir-faire qui présupposent des savoirs, on sait qu'ils appliquent des connaissances et des techniques qu'ils n'ont pas produites. Dans le domaine de la médecine, la distinction est tranchante entre un médecin (un praticien, comme on dit) et un chercheur (producteur de connaissances en biologie humaine).

Cette clôture des institutions scientifiques n'est peut-être pas une clôture comme les autres. Pour aller plus loin, nous allons essayer de cerner la spécificité de l'activité scientifique en tant que production de connaissances du point de vue des processus de communication qui y sont impliqués.

À cet égard, l'essentiel est de prendre en compte ce que nous appellerons le lien communicationnel. Tout acte de communication, en effet, qu'il soit interpersonnel ou médiatique, produit nécessairement un lien. Ce lien peut préexister à un acte de communication donné, lorsqu'il s'est construit dans le temps (par exemple, dans la communication entre les membres d'une même famille ou bien dans la lecture régulière d'un journal), mais chaque acte actualise d'une manière ou d'une autre le lien, l'active d'une façon spécifique. La production-activation de ce lien nous intéresse particulièrement ici, parce qu'il s'agit d'une dimension structurelle et fondamentale de la communication.

Un émetteur quel qu'il soit (personnel ou institutionnel) ne peut communiquer sans se situer lui-même, et en même temps sans situer le récepteur auquel il s'adresse, par rapport à ce qu'il dit. C'est pourquoi nous parlerons de places, que nous appellerons énonciateur (la place de l'émetteur) et destinataire (la place du récepteur). Un acte de communication est la mise en 
relation entre ces places. Un même émetteur pourra construire des places différentes dans des actes de communication différents, d'où l'importance de bien distinguer l'émetteur de l'énonciateur et le récepteur du destinataire. L'étude de ces places et de leurs relations est un aspect de ce qu'on appelle aujourd'hui, en sciences de la communication, l'analyse de l'énonciation (Fisher et Frankel, 1983 ; Fisher et Véron, 1986 ; Culioli, 1990).

Ces quelques distinctions nous permettront maintenant de mettre un peu d'ordre dans le domaine de la communication associée aux connaissances scientifiques. En simplifiant beaucoup, nous allons caractériser quatre situations de communication où le discours qui y circule porte sur des connaissances scientifiques. La nature de ces situations est déterminée par le statut des énonciateurs et des destinataires.

Dans les deux premières, il existe une hypothèse forte de symétrie entre énonciateur et destinataire : ils sont à certains égards fondamentaux comparables, égaux, même si cette hypothèse n'opère pas de la même manière dans les deux cas. Ces deux situations sont endogènes aux institutions scientifiques, elles se produisent à l'intérieur de celles-ci.

\section{Communication endogène intra-disciplinaire}

Dans ce premier cas l'énonciateur et le destinataire sont placés dans la situation à titre de scientifiques travaillant dans un même secteur d'une même discipline. Par exemple, un physicien travaillant dans le domaine des hautes énergies présente de nouveaux résultats d'expériences dont il a été le responsable, à d'autres spécialistes des hautes énergies, dans le cadre d'un congrès (ou sous la forme d'un article dans une revue spécialisée). C'est le cas extrême d'homogénéité et de clôture du circuit communicationnel.

Cette situation se caractérise par un certain nombre de présupposés :

1) l'énonciateur et le destinataire s'auto-définissent comme producteurs de connaissances dans un même domaine scientifique. La situation est donc structurée par une hypothèse forte de symétrie entre les deux positions.

2) Cette symétrie a une implication bien précise : elle est l'une des principales raisons qui justifie l'acte de communication et légitime sa mise en œuvre. Autrement dit: l'une des principales raisons pour lesquelles l'énonciateur est motivé à communiquer avec le destinataire est le statut de producteur de connaissances de ce dernier dans le même domaine. L'énonciateur peut communiquer, sans s'attendre à la moindre rupture institutionnelle, parce qu'il est luimême reconnu par le destinataire comme producteur de connaissances dans le domaine en question.

3) Cette dernière remarque montre bien qu'une autre implication de la symétrie est une hypothèse sur la comparabilité de compétences de l'énonciateur et du destinataire. Dans des situations de ce type, le destinataire sera amené à actualiser cette comparabilité en exerçant 
l'autorité qu'elle comporte : il posera des questions, soulèvera éventuellement des objections, fera différentes sortes de remarques.

Au sein des institutions scientifiques, des situations de ce type ont lieu de façon permanente. Nous avons affaire ici en réalité à une famille de situations de communication, qui comporte des multiples variantes. Nous ne rentrerons pas dans les détails, car ce type de communication n'est pas, en tant que telle, l'objet de cet article. Donnons un seul exemple : un astrophysicien pourra tout naturellement assister à l'exposé de notre spécialiste en physique des hautes énergies. Mais la relation entre sa compétence comme destinataire et celle de l'énonciateur, n'est pas tout à fait de même nature que la relation entre la compétence de l'énonciateur et celle de collègues « immédiats », qui travaillent sur les mêmes appareils que lui.

\section{Communication endogène inter-disciplinaire}

Ce deuxième type de situation est caractéristique de la recherche qui met en contact différents champs disciplinaires à l'intérieưr des institutions scientifiques. La situation est donc endogène comme le cas précédent, mais l'acte de communication implique le passage de frontières entre disciplines. C'est le cas lorsqu'un physicien en physique de particules communique avec un biochimiste, ou lorsqu'un paléontologue communique avec un historien.

Les présupposés qui structurent ce type de situation peuvent être présentés de la façon suivante :

1) une équivalence entre énonciateur et destinataire en tant que producteurs de connaissances scientifiques est toujours postulée.

2) Cette équivalence opère comme justification et légitimation de l'acte de communication.

3) En revanche, il n'y a plus d'hypothèse de symétrie quant aux compétences respectives. Ce qui va affecter certaines modalités des processus de communication et les différencier du cas précédent.

Les deux autres types de situations sont ceux qui nous intéressent directement dans cet article : ils nous font sortir des institutions scientifiques. Leur caractéristique commune est l'hypothèse contraire à celle de la symétrie qui structure, sous deux formes différentes, les deux situations précédemment décrites. Ici, il y a une hypothèse d'asymétrie, de différence, entre l'énonciateur et le destinataire. Ce sont des situations de communication fondées sur la complémentarité et non pas sur la symétrie (Bateson, 1973). La différence entre les deux est que l'une d'elles est endogène comme les précédentes, et l'autre est exogène.

\section{Communication endogène trans-scientifique}

Avec ce type de situation, nous entrons déjà dans ce qu'on appelle souvent par ailleurs la « vulgarisation scientifique ». Ses principales caractéristiques peuvent être décrites comme suit : 
1) l'énonciateur s'auto-définit comme scientifique, comme producteur de connaissances. L'acte de communication a donc son point d'origine à l'intérieur des institutions scientifiques, d'où la qualification d'endogène que nous utilisons.

2) Le destinataire, comme nous l'avons déjà indiqué, est défini par différence : c'est parce qu'il n'est pas un scientifique que l'énonciateur s'adresse à lui.

3) C'est cette différence qui fonde la justification et la légitimité de l'acte de l'énonciateur, c'est parce qu'il a une certaine compétence dans un domaine scientifique, et que le destinataire ne l'a pas, qu'il prend la parole.

De nombreux ouvrages de chercheurs prestigieux écrits, comme on dit, pour le grand public sont un bon exemple de ce type de situation. L'intervention d'un scientifique dans un magazine de télévision est un autre exemple.

\section{Communication exogène sur la science}

Ce type de situation de vulgarisation scientifique est la plus fréquente dans les médias aujourd'hui. Elle a son origine en dehors des institutions scientifiques. Un exemple serait celui du journaliste de télévision qui explique lui-même un phénomène scientifique en s'adressant directement aux téléspectateurs :

1) ni l'énonciateur ni le destinataire ne sont définis comme producteurs de connaissances.

2) La situation comporte cependant, comme la précédente, une hypothèse de complémentarité : c'est la différence entre l'énonciateur et le destinataire qui nourrit la justification et la légitimation de l'acte.

3) On peut se demander ce qui fonde cette différence, étant donné qu'il n'y a pas ici en jeu une compétence légitimée par les institutions scientifiques elles-mêmes. En tout cas, l'énonciateur se construit plus ou moins explicitement comme possédant plus de connaissances que le destinataire auquel il s'adresse.

Nous venons de caractériser quatre types de situations de communication qui concernent, du plus près (type 1) au plus lointain (type 4) les connaissances scientifiques. Prenons à leur égard quelques précautions.

Tout d'abord, elles ne sont certainement pas les seules situations possibles. Les types 1 et 2 , nous l'avons déjà dit, comportent de multiples variantes. Le type 3 aussi, surtout en raison de la spécificité des différents supports médiatiques : écrire un livre et être invité à une émission de télévision sont, pour un scientifique, deux situations qui comportent des contraintes extrêmement différentes. Une multiplicité de configurations communicationnelles peuvent être associées au type 4 .

Deuxièmement, rappelons l'importance de distinguer entre l'émetteur et l'énonciateur, d'un côté, et entre le destinataire et le récepteur d'un autre côté. Beaucoup de téléspectateurs 
d'une émission de vulgarisation du type 4 peuvent être des producteurs de connaissances : après tout, rien n'interdit à un physicien, un chimiste ou un neurobiologiste de regarder la télévision. N'empêche que l'émission en question ne les interpelle pas à ce titre. Ils sont, en tant que récepteurs, des producteurs de connaissances, mais en tant que destinataires de cette émission (s'ils décident de la regarder), ils ne le sont pas.

Troisièmement, il y a des actes de communication qui peuvent comporter la construction de plusieurs destinataires différents, ce qui rend ces actes quelque part hybrides, en les faisant appartenir à deux de nos types à la fois. Une hybridation caractéristique est celle entre les types 2 et 3. Les ouvrages de Gerald Edelman, illustre neurobiologiste, sur le fonctionnement du cerveau me paraissent un très bon exemple de cette hybridation. D'une part, ce sont des livres endogènes, adressés à un destinataire cultivé mais qui n'est certainement pas interpellé en tant que producteur de connaissances scientifiques (type 3). D'autre part, ils s'adressent aussi à des producteurs de connaissances dans d'autres disciplines que la neurobiologie (des psychologues, des chercheurs en sciences cognitives, des philosophes...) qui peuvent s'y intéresser en fonction de leur propre discipline et compte tenu que l'on s'adresse à eux en tant que non-spécialistes en neurobiologie. Ce destinataire-ci renvoie plutôt à notre type 2 .

Même si de tels hybrides peuvent exister, il est nécessaire de distinguer clairement tous ces différents discours autour de la science et de ne pas confondre l'activité discursive de production de savoir - destinée aux autres membres d'une communauté scientifique - et les discours sur les conditions de cette production ou sur ses résultats - qui eux s'adressent à d'autres secteurs de la société. Ce n'est que de cette façon que nous pourrons comprendre leur place parmi l'ensemble des discours sociaux, le sens qu'ils peuvent prendre pour tel ou tel acteur et leur rôle dans un fonctionnement démocratique de la société.

RÉFÉRENCES BIBLIOGRAPHIQUES

Bateson, G., Steps to an Ecology of Mind, Londres, Paladin Granada, 1973 ; en français : Vers une écologie de l'esprit, Paris, Éditions du Seuil, 1978.

Benveniste, É., Problèmes de linguistique générale, tome 2, Paris, Gallimard, 1974.

Culoli, A., Pour une Linguistique de l'énonciation, Paris, Ophrys, 1990.

FisCher, S., FrAnKel, J. J., (éd.), « Linguistique, énonciation : aspects et détermination », Connaissance et Langage, 7 , 1983.

FisChER, S., VÉron, É., « Théorie de l'énonciation et discours sociaux », Études de Lettres, 71, 1986.

VÉron, É., Construire l'événement : les médias et l'accident de Three Mile Island, Paris, Éditions de Minuit, 1981. 